\title{
Compressively-strained GaSb nanowires with core-shell heterostructures
}

\author{
Zhongyunshen Zhu ${ }^{1}(\varangle)$, Johannes Svensson ${ }^{1}$, Axel R. Persson ${ }^{2,3}$, Reine Wallenberg ${ }^{2,3}$, Andrei V. Gromov ${ }^{4}$, \\ and Lars-Erik Wernersson ${ }^{1}$
}

${ }^{1}$ Department of Electrical and Information Technology, Lund University, Box 118, 22100 Lund, Sweden

${ }^{2}$ Centre for Analysis and Synthesis, Lund University, Box 124, 22100 Lund, Sweden

${ }^{3}$ NanoLund, Lund University, Box 118, 22100 Lund, Sweden

${ }^{4}$ EaStCHEM, School of Chemistry, University of Edinburgh, Edinburgh EH9 3FJ, UK

(C) The Author(s) 2020

Received: 19 March 2020 / Revised: 27 April 2020 / Accepted: 18 May 2020

\begin{abstract}
GaSb-based nanowires in a gate-all-around geometry are good candidates for binary p-type transistors, however they require the introduction of compressive strain to enhance the transport properties. Here, we for the first time demonstrate epitaxial GaSb$\mathrm{GaAs}_{x} \mathrm{Sb}_{1-x}$ core-shell nanowires with a compressively strained core. Both axial and hydrostatic strain in GaSb core have been measured by X-ray diffraction (XRD) and Raman scattering, respectively. The optimal sample, almost without plastic relaxation, has an axial strain of $-0.88 \%$ and a hydrostatic strain of $-1.46 \%$, leading to a noticeable effect where the light hole band is calculated to be $33.4 \mathrm{meV}$ above the heavy hole band at the $\Gamma$-point. This valence band feature offers more light holes to contribute the transport process, and thus may provide enhanced hole mobility by reducing both the interband scattering and the hole effective mass. Our results show that lattice-mismatched epitaxial core-shell heterostructures of high quality can also be realized in the promising yet demanding GaSb-based system.
\end{abstract}

KEYWORDS

compressive strain, GaSb-GaAs $\mathrm{Sb}_{1-x}$, core-shell, nanowires, heterostructure, $\mathrm{p}$-type transistors

\section{Introduction}

Metal-oxide-semiconductor field-effect transistors (MOSFETs) based on III-V semiconductor nanowires (NWs) with vertical architecture and gate-all-around geometry have sparked intensive interests [1-5] due to high mobilities and injection velocities in III-V materials [6] and good electrostatics enabling aggressive gate length scaling [7]. Among the III-V binary compounds suitable for MOSFETs, GaSb has been a promising candidate for p-type NW transistors as complementary metal-oxidesemiconductor (CMOS) devices [2, 4, 8] due to its high hole mobility [9-11]. Recently, many successful and important investigations, including growth direction control [12] and reduced doping level [13], have been made to enhance the hole mobility of GaSb NWs. The introduction of compressive strain in GaSb, however, is required to increase the hole transport properties further [14]. In the case of planar transistors, latticemismatched $\mathrm{GaSb} / \mathrm{AlAsSb}$ heterostructures with a biaxial strain of $-1 \%$ to $-3 \%$ (negative values denote compressive strains while positive ones denote tensile strains) in GaSb layer have been already demonstrated to increase the mobility by over twice in contrast to that of the bulk $[15,16]$. Therefore, epitaxial heterostructures can provide a considerable option to highly strain GaSb NWs as well. However, most reports of lattice-mismatched epitaxy related to GaSb NWs, such as InAs/GaSb $[8,17,18]$, and GaAs/GaSb $[19,20]$, focused on axial heterostructures in which the strain is only localized near the interface due to the large aspect ratio of NWs [21].

A lattice-mismatched core-shell structure can accommodate uniform strain in the core along the growth direction, which has been suggested by both simulations [22, 23] and recent experiments of GaAs-InGaAs [24, 25] and InAsP-InP core-shell NWs $[26,27]$. The strain effects in NWs also exhibited a larger axial strain than other strain components, indicating a nearuniaxial strain distribution in the core, which can not only lift the degeneracy of valence bands but provide a small effective mass along the transport direction due to a light-hole (LH) dispersion instead of heavy-hole $(\mathrm{HH})$ dispersion adjacent the $\Gamma$-point $[28,29]$. Thus, the mobility can be efficiently enhanced by reducing both the interband scattering and the hole effective mass [29]. In the case of GaSb NWs, however, there are only few reports on electrical properties of GaSb-InAs [30, 31] and GaSb-InAs $0.9 \mathrm{Sb}_{0.1}$ [32] core-shell NWs where the strain in the core is limited by small lattice misfit and large core diameters. Although GaAs-GaSb core-shell NWs were demonstrated, the GaSb shell was plastically relaxed due to unbearably high misfit between GaAs and GaSb [33]. To transfer more strain into GaSb core NWs with the same shell thickness and further to enhance the mobility as well as benefit the electrostatics of transistors by reducing the total core-shell NW diameter, a thin GaSb core is essential [34]. Nevertheless, differing from the mature control in epitaxy of III-As and III-P NWs, high 
quality growth of III-Sb NWs still faces difficulties including the limitation in shrinking diameters [35] and sensitive nucleation condition because of Gibbs-Thompson effects in Sb precursors [36]. Therefore, the knowledge about strain engineering in GaSb-based NWs is very restricted in spite of its significant influence on potential improvement in electrical properties.

In this work, we for the first time demonstrate compressivelystrained GaSb NWs with a GaSb-GaAs $\mathrm{Sb}_{1-x}$ core-shell structure epitaxially grown on pre-patterned $\mathrm{Si}$ substrates by metalorganic vapor phase epitaxy (MOVPE). Large arrays with an area of $1.5 \mathrm{~cm} \times 3 \mathrm{~cm}$ were utilized to enable highly homogeneous sizes and good positioning in NWs. The GaSb core and $\mathrm{GaAs}_{x} \mathrm{Sb}_{1-x}$ shell were successively grown on an InAs stem whose growth was initialized using the Au-assisted vaporliquid-solid process (see details in Methods). The misfit between $\mathrm{GaSb}$ and $\mathrm{GaAs}_{x} \mathrm{Sb}_{1-x}$ ranges $1.5 \%-3.9 \%$ while the highest axial strain reaches $-0.92 \%$ by reducing GaSb core diameter down to $42 \mathrm{~nm}$.

\section{Results and discussion}

The morphology of InAs/GaSb as well as InAs/GaSb-GaAs $\mathrm{Sb}_{1-x}$ core-shell NWs were examined by scanning electron microscopy (SEM) (Figs. 1(a) and 1(b)). By comparing the diameters of the different segments for increasing $\mathrm{GaAs}_{x} \mathrm{Sb}_{1-x}$ shell growth time (Fig. 1(c)), we conclude that a significant shell growth only occurs on the sidewalls of the zincblende GaSb segment and no measurable shell grows on the wurtzite InAs stem due to the lower surface energy [37]. The diameter of the core-shell NWs increases almost linearly with the shell growth time, with a stable growth rate of $\sim 0.44 \AA$ s. During the radial shell growth, a short axial GaAsSb segment on top of the NW is also grown from the Au particle.

A transmission-electron microscope (TEM) image of a NW with core diameter $d_{\text {core }}=50 \mathrm{~nm}$ and shell thickness $t_{\text {shell }}=8$ $\mathrm{nm}$ is shown in Fig. 1(d), combined with a corresponding diffraction pattern, which indicates [111] as the NW growth direction and $(1 \overline{1} 0)$ as the upper sidewall facet in agreement with our earlier studies [19]. Both axial and shell growth of $\mathrm{GaAs}_{x} \mathrm{Sb}_{1-x}$ were visible simultaneously in the TEM as shown in Fig. 1(d) and separated by the dashed lines, in agreement with the SEM inspection in Fig. 1(b). Scanning TEM (STEM) along with X-ray energy dispersive spectroscopy (XEDS) is used to determine the As composition in both the shell and the axial segment displayed in Fig. 1(e). The elemental distribution of $\mathrm{Ga}, \mathrm{Sb}$ and As was obtained by XEDS mapping of the center of the STEM image, distinguishing the boundary of the core and shell. Meanwhile, a radial linescan gives $x=\sim 0.4$ in the $\mathrm{GaAs}_{x} \mathrm{Sb}_{1-x}$ shell $(20 \%$ As in atomic percentage of the sum of $\mathrm{Ga}$, As and $\mathrm{Sb}$ ), which is close to the $x=0.39$ obtained for the axial $\mathrm{GaAs}_{x} \mathrm{Sb}_{1-x}$ segment (see Fig. S1(a) in Electronic Supplementary Material (ESM), Section S1).

X-ray diffraction (XRD) measurements with (111) $\omega / 2 \theta$-scans were employed for probing the axial strain in the GaSb core. As sketched in Fig. 2(a), A large $\omega$-offset $\Delta \omega=0.8^{\circ}$ was utilized for suppressing the diffraction signal from the bulk substrate and planar growth (see Fig. S4(a) in the ESM, Section S3) owing to the fact that NWs exhibit broad peaks along the $\omega$ axis [38]. Figure 2(b) presents the XRD curves of bare GaSb NWs and GaSb-GaAs $0.33 \mathrm{Sb}_{0.67}$ core-shell NWs with $t_{\text {shell }}=9.2 \mathrm{~nm}$. For clarity, we set the peak positions of the InAs buffer layer (zincblende) $\left(2 \theta_{\text {sub }}\right)$ to $0^{\circ}$ to obtain the relative peak positions $\left(2 \theta-2 \theta_{\text {sub }}\right)$. The strongest peaks of both XRD spectra account for $\mathrm{GaSb}$ since it has a much larger volume as compared to the InAs and GaAsSb segments. The GaSb peak of the core-shell NW corresponds to an axial strain $\left(\varepsilon_{z z}\right)$ of $-0.92 \%$ in the core. For the sample in Fig. 1(d), the axial strain in the GaSb core determined from XRD agrees well with the strain obtained from (111) planar distance measured in TEM by doing selective fast-Fourier transformation of the GaSb core (see Fig. S1(c) in the ESM, Section S1). Thus, the $\varepsilon_{z z}$ determined from XRD is reasonable. The shell As composition was determined by the
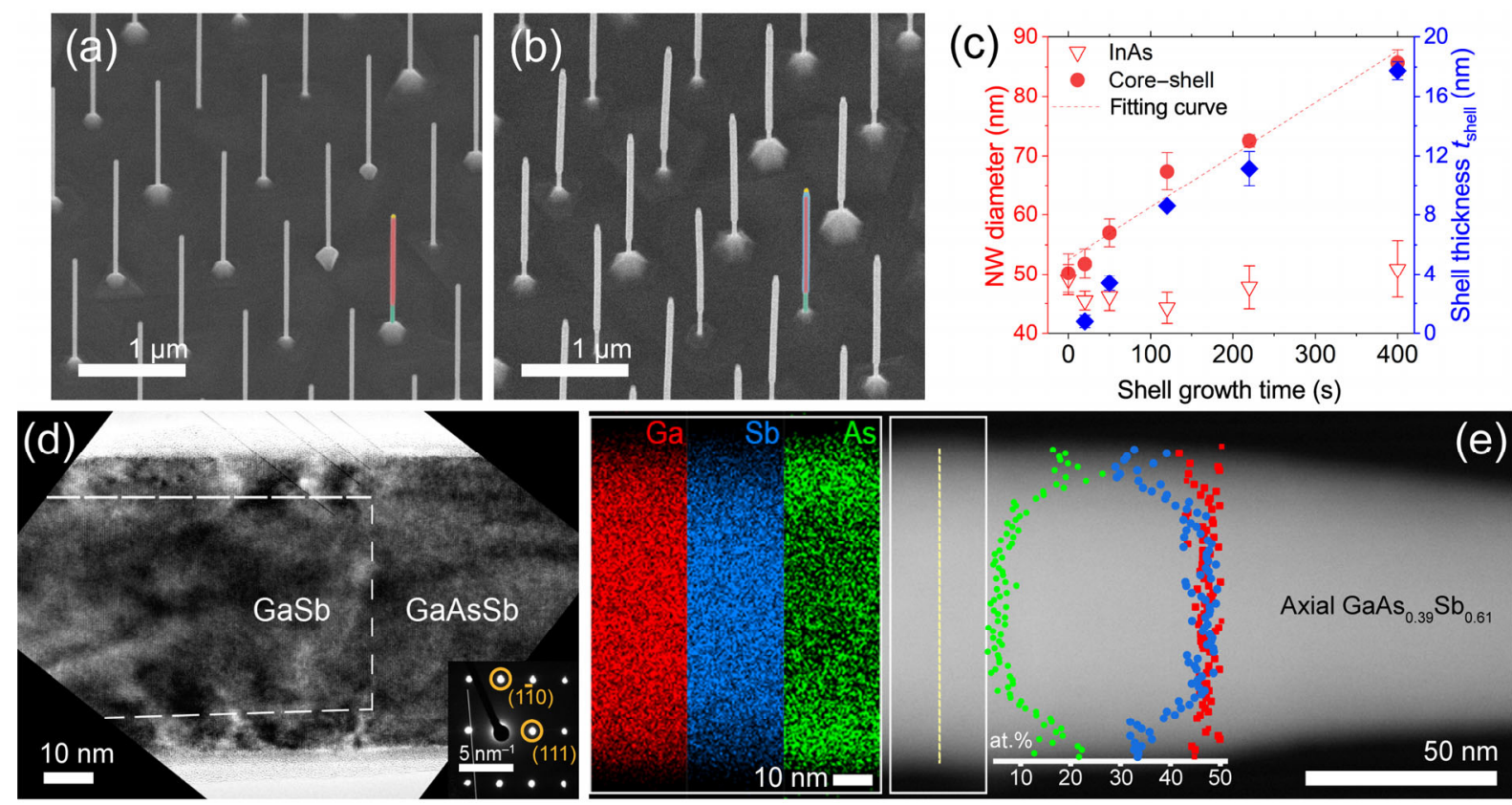

Figure 1 SEM images with $30^{\circ}$-tilted view of NWs grown with Au size of $32 \mathrm{~nm}$ : (a) GaSb NWs; (b) GaSb-GaAs ${ }_{0.45} \mathrm{Sb}_{0.55}$ core-shell NWs with the shell growth time of $120 \mathrm{~s}$. False colors indicate different segments in NWs. (c) Statistical measurements of NW diameters. GaAs $\mathrm{Sb}_{1-x}$ shell can be verified grown only on GaSb sidewalls. (d) TEM image of a core-shell NW with $d_{\text {core }}=50 \mathrm{~nm}$ and $t_{\text {shell }}=8 \mathrm{~nm}$ shows both GaSb and GaAs $x \mathrm{Sb}_{1-x}$ (both the shell and axial segment) segments separated by dashed lines. The inset shows the corresponding diffraction pattern of the NW, indicating the NW growth direction and sidewall facet. (e) XEDS mapping (white rectangle area) and linescan (yellow dashed line) give both the elemental distribution and atomic compositions, respectively. The axial As composition of $\mathrm{GaAs}_{x} \mathrm{Sb}_{1-x}$ was verified using both XRD and XEDS in Fig. S1, Section S1 in the ESM. 

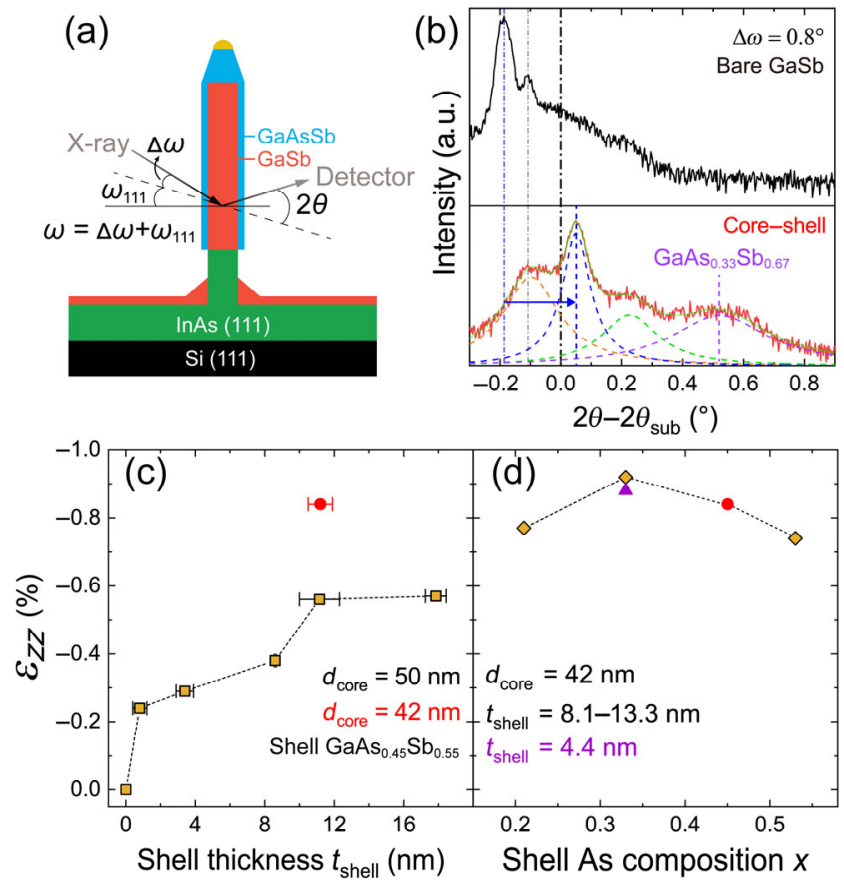

Figure 2 (a) Schematics of XRD measurements of core-shell NWs by offsetting the value of $\omega$. (b) (111) $\omega / 2 \theta$ scans of GaSb core NWs and GaSb-GaAs $0.33 \mathrm{Sb}_{0.67}$ core-shell NWs with $d_{\text {core }}=42 \mathrm{~nm}$ and $t_{\text {shell }}=9.2 \mathrm{~nm}$. $\Delta \omega=0.8^{\circ}$ was selected to eliminate the interference from the planar signals. The blue and black dashed vertical lines denote unstrained GaSb and zincblende InAs peak positions, respectively. (c) and (d) show the axial strain $\varepsilon_{z z}$ extracted from XRD as a function of $t_{\text {shell }}$ and As composition, respectively. The data point of $t_{\text {shell }}=8.6 \mathrm{~nm}$ has an error bar of $0.05 \mathrm{~nm}$ (too narrow to be seen) along $x$-axis in (c). Red dots in (c) and (d) denote the same sample that is shown in the bottom of (b). Lorentzian curves were used to fit the line-shape of the core-shell NW XRD result in (b). The growth rate of the shell was found to be slightly different when varying the As composition but remaining the same growth time of $220 \mathrm{~s}$ in (d).

diffraction peak of unstrained axial GaAsSb, which is well-fitted by the purple Lorentzian curve in Fig. 2(b). The As composition in axial GaAsSb was validated by XEDS results, as discussed in the ESM, Section S1, where $x=0.387$ was obtained from XRD measurement (see Fig. S1(b) in the ESM) for the sample given in Fig. 1(e), in good agreement with 0.39 obtained from EDX. Thus, the shell As composition can be determined from the unstrained axial GaAss $\mathrm{Sb}_{1-x}$ segment which is detectable in the XRD. A weak signal observed at $-0.11^{\circ}$ in both samples corresponds to unstrained wurtzite InAs [39]. We speculate that the peak at $0.224^{\circ}$ (green fitting curve) might originate from the strained $\mathrm{GaAs}_{x} \mathrm{Sb}_{1-x}$ shell, corresponding to an axial tensile strain of $1.15 \%$ but the peak position changes anomalously when varying $t_{\text {shell }}$ or shell As composition (discussed in the ESM, Section S3).

The axial strains, $\varepsilon_{z z}$, determined from XRD peak positions for GaSb are summarized in Figs. 2(c) and 2(d) for samples with varying $t_{\text {shell }}$ and shell As composition, respectively. For NWs with $\mathrm{GaAs}_{0.45} \mathrm{Sb}_{0.55}$ shell, $\varepsilon_{z z}$ increases in the $t_{\text {shell }}$ range from 0.8 to $11.2 \mathrm{~nm}$ and saturates with the value of $-0.57 \%$ at $t_{\text {shell }}=11.2 \mathrm{~nm}$ (Fig. 2(c)). Moreover, $\varepsilon_{z z}$ increases significantly when reducing $d_{\text {core }}$ down to $42 \mathrm{~nm}$ but with the same shell thickness of $t_{\text {shell }}=11.2 \mathrm{~nm}$ (red dot). In Fig. 2(d), the values of $\varepsilon_{z z}$ are larger for these samples with a thinner core diameter of $d_{\text {core }}=42 \mathrm{~nm}$, as compared to those with varying $t_{\text {shell. }}$ In addition, increasing the As composition up to 0.33 gradually raises the axial strain in the GaSb core, reaching a maximum strain of $-0.92 \%$. This value is close to the $-1 \%$ uniaxial strain that is theoretically calculated by density functional theory to decrease the hole effective mass down to $0.05 m_{0}$ along [111] in $\mathrm{GaSb}$ [40]. This value is 8 times smaller than the unstrained $\mathrm{HH}$ mass of $0.4 m_{0}$, and is thus expected to lead to a mobility improvement [9]. However, $\varepsilon_{z z}$ drops when incorporating more As in the $\mathrm{GaAs}_{x} \mathrm{Sb}_{1-x}$ shell, suggesting that an optimal As composition close to $x=0.33$ gives the highest strain for $t_{\text {shell }}=8.3-13.3 \mathrm{~nm}$, which is similar to results reported for GaAs-InGaAs core-shell NWs [24]. The reason for this result can be interpreted as different plastic relaxation in the samples with different As compositions, which will be discussed later. Surprisingly, the sample with $4.4 \mathrm{~nm} \mathrm{GaAs} \mathrm{n}_{0.33} \mathrm{Sb}_{0.67}$ shell exhibits a high axial strain of $-0.88 \%$, which is very close to the highest strain $(-0.92 \%)$ in the sample with $9.2 \mathrm{~nm} \mathrm{GaAs} 0.33 \mathrm{Sb}_{0.67}$ shell. A significant suppression of plastic relaxation along [111] direction was found in the sample with $4.4 \mathrm{~nm} \mathrm{GaAs} 0.33 \mathrm{Sb}_{0.67}$ shell, accounting for the reason of this phenomenon, which will be discussed in detail later. The shell thickness of samples in this series varies due to that different shell As compositions give different growth rates (see Section S2 in the ESM). Although a different strain distribution as a single function of the As composition is expected, the conclusion that GaSb core reaches the maximum axial strain with a $\mathrm{GaAs}_{0.33} \mathrm{Sb}_{0.67}$ shell is still valid since the strain decreases when increasing both the shell thickness and the As composition.

High-resolution TEM (HRTEM) was used to estimate the density of edge dislocation loops (loop dislocations) originating from the large misfit of the core and shell, which can introduce the plastic relaxation along [111] in the core. Figure 3(a) reveals three local dislocations (black arrows) near the interface of the core and shell. One additional atomic plane is observed in the shell (Fig. 3(b)), leading to a reduced lattice mismatch along the $z$-axis between the GaSb core and $\mathrm{GaAs}_{x} \mathrm{Sb}_{1-x}$ shell. No matter the direction of the loop dislocation, there is always a $z$-component of Burgers vector involved [41]. Hence, we can attribute the local dislocations seen in TEM to loop dislocations perpendicular to the NW growth axis, sketched in Fig. 3(c). The simulated axial strain field in a core-shell NW with the same geometry and shell As composition as shown in Fig. 3(a) is presented in Fig. 3(d), showing $\varepsilon_{z z}^{\prime}=-0.86 \%$ without any dislocations (only elastic relaxation occurs), whereas the experimental value measured in XRD gives $\varepsilon_{z z}=-0.11 \%$. Here we define the axial strain reduction in percentage as the dislocation-induced plastic relaxation $R_{\mathrm{p}}=\left(\varepsilon_{z z}^{\prime}-\varepsilon_{z z}\right) / \varepsilon_{z z}^{\prime}$, resulting in $87 \%$ plastic relaxation for this sample. This gives a density of $7.7 \times 10^{5} \mathrm{~cm}^{-1}$ assuming a uniform loop dislocation distribution (Fig. 3(a)) (see details in the ESM, Section S4). To reach the experimentally measured strain of $\mathcal{E}_{z z}=-0.11 \%$, loop dislocations with a density of $7.9 \times 10^{5} \mathrm{~cm}^{-1}$ had to be included in the simulation (Fig. 3(e)) (see Method). This density is in good agreement with that observed in TEM. Therefore, we can confirm that the axial strain reduction is mainly due to plastic relaxation caused by loop dislocations.

Figures 3(f) and $3(\mathrm{~g})$ present the simulation results of $R_{\mathrm{p}}$ as a function of shell thickness and As composition, respectively. The axial strain $\varepsilon_{z z}$ can be written as $\varepsilon_{z z}=\varepsilon_{z z}^{\prime}\left(1-R_{\mathrm{p}}\right)$. For a $\mathrm{GaAs}_{0.45} \mathrm{Sb}_{0.55}$ shell with $t_{\text {shell }}>3.4 \mathrm{~nm}$, a similar $R_{\mathrm{p}}(\sim 70 \%)$ was calculated, so $\varepsilon_{z z}$ is only determined by the elastic strain $\varepsilon_{z z}^{\prime}$ which here mainly depends on $t_{\text {shell. }}$ Thus, $\varepsilon_{z z}$ increases and eventually saturates, the same as reported cases [25, 26]. For $t_{\text {shell }}<3.4 \mathrm{~nm}, R_{\mathrm{p}}$ gradually increases from $20 \%$ to a high level (Fig. 3(f)) that effectively limits the axial strain increasing so that $\varepsilon_{z z}$ increases fast first and slowly afterwards (Fig. 2(c)). In Fig. $3(\mathrm{~g})$, the plastic relaxation $R_{\mathrm{p}}$ increases with the shell As composition. Additionally, both $\varepsilon_{z z}^{\prime}$ and $R_{\mathrm{p}}$ increase when increasing the shell As composition with a similar $t_{\text {shell, }}$ resulting 

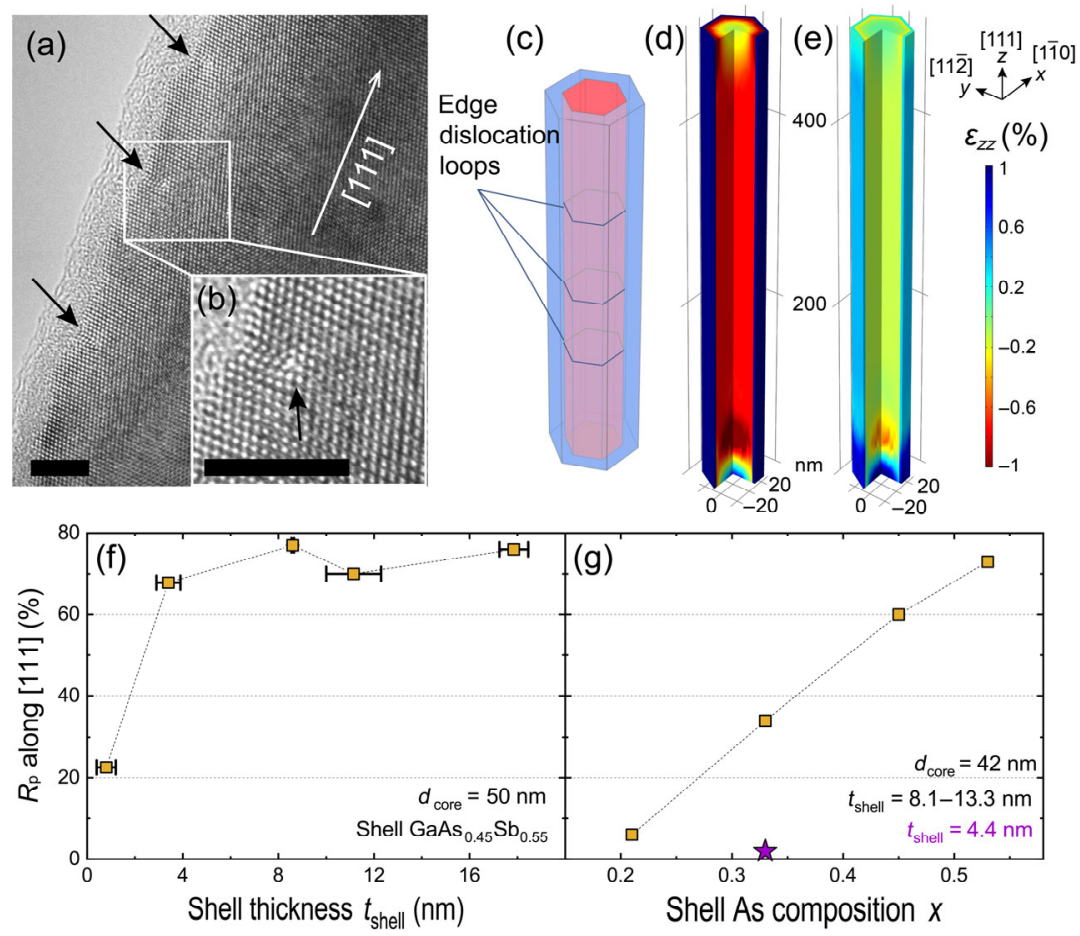

Figure 3 (a) and (b) HRTEM image of a GaSb-GaAs $s_{0.45} \mathrm{Sb}_{0.55}$ core-shell $\mathrm{NW}$ with $t_{\text {shell }}=3 \mathrm{~nm}$ and $d_{\text {core }}=55 \mathrm{~nm}$. The arrows indicate three loop dislocations near the interface of core and shell. The distance of each two dislocations along [111] is $\sim 12.5 \mathrm{~nm}$. The scale bars are $5 \mathrm{~nm}$. (c) Schematic of the loop dislocations. (d) and (e) 3D strain field simulations of a NW with the same geometry as that observed by HRTEM in (a) without and with loop dislocations. The average strain can be regarded as the strain in the middle of the NW due to that the top and bottom edges have a negligible contribute to the total strain if the NW is sufficiently long (500 nm here). The density of loop dislocations in simulation (e) is $7.9 \times 10^{5} \mathrm{~cm}^{-1}$, in agreement with $7.7 \times$ $10^{5} \mathrm{~cm}^{-1}$ which was estimated from HRTEM (a) (see Section S4 in the ESM). The plastic relaxation $R_{\mathrm{p}}=\left(\varepsilon_{z z}^{\prime}-\varepsilon_{z z}\right) / \varepsilon_{z z}^{\prime}$ as a function of $t_{\text {shell }}$ and shell As composition $x$ were simulated in (f) and (g), respectively. The data point of $t_{\text {shell }}=8.6 \mathrm{~nm}$ has an error bar of $0.05 \mathrm{~nm}$ (too narrow to be seen) along $x$-axis in (f). The growth rate of the shell was found to be slightly different when varying the As composition but remaining the same growth time of $220 \mathrm{~s}$ in (g).

in a tradeoff in $\varepsilon_{z z}^{\prime}$ and $R_{\mathrm{p}}$ to emerge a maximum value of $\varepsilon_{z z}$ with an optimal $x=0.33$ in $\mathrm{GaAs}_{x} \mathrm{Sb}_{1-x}$. When shrinking $t_{\text {shell }}$ from 9.2 to $4.4 \mathrm{~nm}$ but remaining the same $\mathrm{GaAs}_{0.33} \mathrm{Sb}_{0.67}$ shell, the drop of $R_{\mathrm{p}}$ from $34 \%$ to $2 \%$ (the purple star in Fig. $3(\mathrm{~g})$ ) balances the elastic strain $\varepsilon_{z z}^{\prime}$ reduction, which leads to a similar $\mathcal{E}_{z z}$ in the core. The significant suppression of plastic relaxation in the sample with $4.4 \mathrm{~nm} \mathrm{GaAs} 0.33 \mathrm{Sb}_{0.67}$ shell can be explained by the critical shell thickness in the lattice-mismatched core-shell structure [42]. For $d_{\text {core }}=42 \mathrm{~nm}$ and a GaAs ${ }_{0.33} \mathrm{Sb}_{0.67}$ shell, we speculate that the critical shell thickness should be slightly smaller than $4.4 \mathrm{~nm}$, indicating almost no presence of plastic relaxation. However, increasing $t_{\text {shell }}$ to $9.2 \mathrm{~nm}$, which is far beyond the critical shell thickness, introduces plastic relaxation to the axial strain, thus no evident strain increase.

Finally, Raman microscopy was performed on individual NWs to enable evaluation of the hydrostatic strain $\left(\varepsilon_{x x}+\varepsilon_{y y}+\right.$ $\left.\varepsilon_{z z}\right)$ of the core-shell NWs, thus calculating the valence band shifts at the $\Gamma$-point. Figure 4(a) presents Raman spectra of a bare GaSb NW and a core-shell NW from the same sample as Fig. 2(b). In the case of the bare GaSb NW, both transverse optical (TO) and longitudinal optical (LO) phonons are clearly observed, with peak positions at 224.8 and $233.3 \mathrm{~cm}^{-1}$, respectively, in good agreement with reported values [43]. For the core-shell NW, however, the LO mode was dramatically suppressed even with a shell as thin as $0.8 \mathrm{~nm}$ (see all Raman spectra in the ESM, Section S5), while the TO mode still has a high intensity at a wavenumber of $230.2 \mathrm{~cm}^{-1}$ as shown in Fig. 4(a) bottom. By using Lorentzian fitting, the LO phonon peak can be estimated at $242.6 \mathrm{~cm}^{-1}$. Moreover, with the addition of the shell, a third peak appears at $223.6 \mathrm{~cm}^{-1}$ (GaSb-like TO) which is close to the unstrained one probably coming from the relaxed part in the GaSb core. Due to its high intensity in core-shell NWs we focus our analysis on the shift of the GaSb TO phonons as a function of $t_{\text {shell }}$ and shell As composition (Figs. 4(b) and 4(c)). Without the consideration of any other potential contributions [44], the Raman shift of the GaSb TO peaks is attributed exclusively to the strain in the core. Besides, no peak splitting for GaSb TO phonons in any sample was found, and thus the hydrostatic strain can be approximately deduced as a linear function of the relative Raman shift $\left(\Delta \Omega / \Omega_{\mathrm{TO}}\right)$ of $\mathrm{GaSb}$ TO peaks $[34,45]$ as

$$
\varepsilon_{\text {hyd }}=\varepsilon_{x x}+\varepsilon_{y y}+\varepsilon_{z z} \approx 1 / \gamma_{\mathrm{TO}} \cdot \Delta \Omega / \Omega_{\mathrm{TO}}
$$

where $\gamma_{\mathrm{TO}}=1.33$ is Grüneisen parameter [43], describing the hydrostatic deformation potential of GaSb TO phonons. The hydrostatic strains are presented in Figs. 4(d) and 4(e) followed by Eq. (1). As also observed using XRD, the core becomes more strained with increasing $t_{\text {shell, }}$, whereas the hydrostatic strain $\varepsilon_{\text {hyd }}$ saturates at $-1.28 \%$ for $t_{\text {shell }} \geq 11.2 \mathrm{~nm}$ and $d_{\text {core }}=50 \mathrm{~nm}$. Similarly, for $d_{\text {core }}=42 \mathrm{~nm}$, the hydrostatic strain in the core reaches $-1.64 \%$, an increase of $27 \%$ with respect to the strain in the case of $d_{\text {core }}=50 \mathrm{~nm}$. When varying the shell As composition, the hydrostatic strain exhibits the same feature as the axial strain, which gives a maximum $\mathcal{E}_{\text {hyd }}=-1.91 \%$ in the sample with the $9.2 \mathrm{~nm} \mathrm{GaAs}{ }_{0.33} \mathrm{Sb}_{0.67}$ shell. The same conclusion can be drawn when considering the shell thickness variation.

Combining the axial strain with the hydrostatic strain in the core, we are able to deduce the sum of the radial strain $\varepsilon_{x x}+\varepsilon_{y y}$, and thus estimate the band edge of the strained GaSb core. The band edge depends on both the hydrostatic strain $\varepsilon_{\text {hyd }}$ and the strain component $\varepsilon_{\mathrm{vbs}}=\varepsilon_{z z}-0.5 \cdot\left(\varepsilon_{x x}+\varepsilon_{y y}\right)$ (here, the subscript "vbs" denotes the valence band splitting since this strain component only affects the LH maximum shift) [46]. Table 1 lists the calculated results of the difference between the 

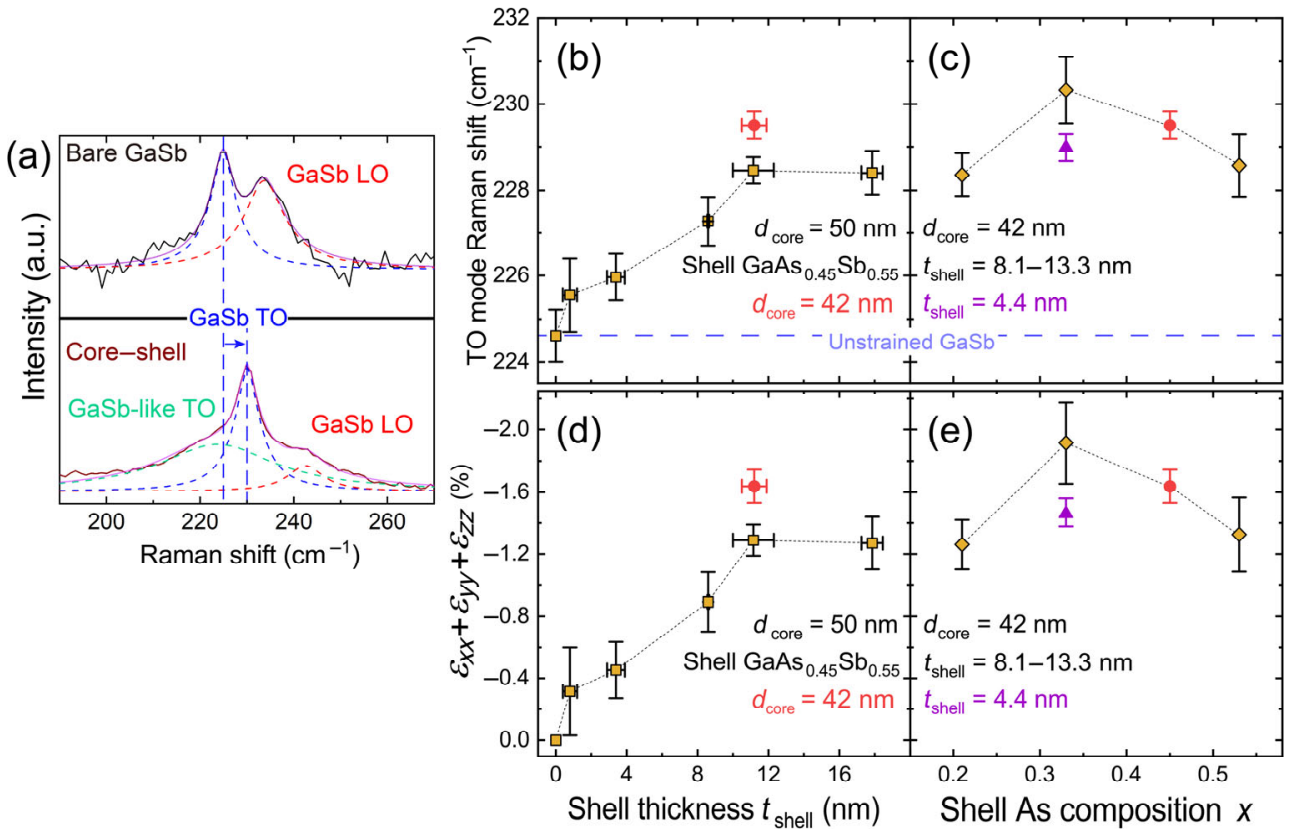

Figure 4 (a) Raman spectrum of a core-shell NW (bottom) from the same sample as in Fig. 2(b) and a spectrum of a bare GaSb NW (top). (b) and (c) show the TO mode shift of the GaSb core with varying the shell thickness $t_{\text {shell }}$ and shell As composition $x$, respectively. (d) and (e) show the corresponding extracted hydrostatic strain. Red dots in ((b) and (c)) or ((d) and (e)) correspond to the same sample. Lorentzian fitting was used for Raman curves to interpret the different scattering modes. The growth rate of the shell was found to be slightly different when varying the As composition but remaining the same growth time of $220 \mathrm{~s}$ in (e).

Table 1 Various strain components extracted from XRD and Raman measurements (Figs. 2(d) and 4(e)), the energy difference between LH and HH maximum $\left(E_{\mathrm{LH}}-E_{\mathrm{HH}}\right)$ and bandgap $\left(E_{\mathrm{g}}\right)$ of strained GaSb NW calculated by the band edge deformation potential theory are listed

\begin{tabular}{ccccccc}
\hline Shell type & $t_{\text {shell }}(\mathrm{nm})$ & $\varepsilon_{z z}$ & $\varepsilon_{\text {hyd }}$ & $\varepsilon_{\mathrm{vbs}}$ & $E_{\mathrm{LH}}-E_{\mathrm{HH}}(\mathrm{meV})$ & $E_{\mathrm{g}}(\mathrm{eV})$ \\
\hline No shell & 0 & 0 & 0 & 0 & 0 & 0.72 \\
$\mathrm{GaAs}_{0.33} \mathrm{Sb}_{0.67}$ shell & 9.2 & $-0.92 \%$ & $-1.91 \%$ & $-0.43 \%$ & 23.9 & 0.85 \\
$\mathrm{GaAs}_{0.33} \mathrm{Sb}_{0.67}$ shell & 4.4 & $-0.88 \%$ & $-1.46 \%$ & $-0.59 \%$ & 33.4 & 0.81 \\
\hline
\end{tabular}

$\mathrm{LH}$ and $\mathrm{HH}$ maxima $\left(E_{\mathrm{LH}}-E_{\mathrm{HH}}\right)$ and the bandgap $\left(E_{\mathrm{g}}\right)$ of the strained GaSb core (see Methods), resulting from different strain components. By shrinking $t_{\text {shell }}$ from 9.2 to $4.4 \mathrm{~nm}, \varepsilon_{z z}$ keeps similar while $\varepsilon_{\text {hyd }}$ drops much, leading to an increasing value of $E_{\mathrm{LH}}-E_{\mathrm{HH}}$ up to $33.4 \mathrm{meV}$.

In fact, the splitting between the $\mathrm{LH}$ and $\mathrm{HH}$ maxima mainly depends on $\varepsilon_{\mathrm{vbs}}$ [46], with a more negative $\varepsilon_{\mathrm{vbs}}$ resulting in larger $E_{\mathrm{LH}}-E_{\mathrm{HH}}$, as shown in Table 1 . Hence, it is preferable to increase the axial compressive strain $\varepsilon_{z z}$ but simultaneously decrease the radial compressive strain $\varepsilon_{x x}+\varepsilon_{y y}$ (or $\varepsilon_{\text {hyd }}$ ), thus increasing the valence band splitting to lower the rate of interband scattering, a process usually accompanied by absorption or emission of optical phonons [47]. This means the interband scattering rate for holes at the $\Gamma$-point at the valence band maximum can be drastically lowered as long as the separated energy $E_{\mathrm{LH}}-E_{\mathrm{HH}}$ is greater than the optical phonon energy $h v_{\mathrm{O}}$ [29]. Thanks to the nearly absence of plastic relaxation, the sample with $t_{\text {shell }}=4.4 \mathrm{~nm}$ has similarly high axial strain as the sample with $t_{\text {shell }}=9.2 \mathrm{~nm}$ while a manifest reduction in $\varepsilon_{\text {hyd }}$, thus providing a more significant valence band splitting of $33.4 \mathrm{meV}$ which is greater than the optical phonon energy $h v_{\mathrm{o}} \sim 28 \mathrm{meV}$ with the optical phonon frequency $v_{0} \sim 230 \mathrm{~cm}^{-1}$ extracted from Raman. As a consequence, suppression of interband scattering is expected for this sample.

In addition, the LH band appears at the top of the valence band $\left(E_{\mathrm{LH}}-E_{\mathrm{HH}}>0\right)$, allowing more light holes to contribute to the electrical transport. In the other words, the hole effective mass should be much reduced near the $\Gamma$-point. Also, highly compressive strain along the transport direction [111] can also increase the curvature in the $E$ - $\boldsymbol{k}$ dispersion (band warping), leading to a further reduction in the effective mass of holes along [111]. Both the suppression of the interband scattering and the effective mass lowering along transport axis are therefore expected to improve the hole transport properties in compressively strained GaSb core NWs. However, in the case of biaxial compressive strain in planar GaSb films, although the degeneracy of valence bands is lifted the $\mathrm{HH}$ maximum stays above the LH maximum, resulting in the mobility enhancement mainly due to the valence band splitting that reduces the interband scattering between the valence bands, rather than slight reduction in the effective mass due to band warping [48]. Consequently, a very high strain is required to split the valence bands sufficiently to enhance the mobility for biaxial compression. In contrast, the core-shell structure with high axial strain and small hydrostatic strain may more efficiently enhance the mobility than that with biaxial strain in the film. This agrees well with simulation results from Nainani et al. [49] as well as experimental data from Thompson et al. [50] which indicated that the same strain level result in higher mobility in the uniaxial case than in the biaxial one. On the other hand, the bandgap widening due to the compressive strain may benefit the off-state performance of a transistor. More axial compressive strain in GaSb core is likely to further increase the hole mobility. The best option is considered to reduce the core diameter even more, which can significantly increase the critical shell thickness as well as the tolerance of lattice misfit for the same critical shell thickness [42, 51]. However, it is currently challenging to reduce the diameter of 
$<111>$-oriented GaSb NWs grown by MOVPE below $32 \mathrm{~nm}$ [35]. Thus, for a NW with $d_{\text {core }}=32 \mathrm{~nm}$, based on simulation, $-1.08 \%$ axial strain can be achieved (increasing $23 \%$ compared to the case of $d_{\text {core }}=42 \mathrm{~nm}$ ) in the GaSb core without any plastic relaxation by selecting $\mathrm{GaAs}_{0.33} \mathrm{Sb}_{0.67}$ shell and $t_{\text {shell }}=$ $4.4 \mathrm{~nm}$. This structure is expected to further enlarge $E_{\mathrm{LH}}-E_{\mathrm{HH}}$ (up to $52 \mathrm{meV}$ ) and band warping along [111], thus improving the hole mobility further by reducing both the interband scattering and the hole effective mass. Besides, external stress from processing [52] or mechanics [53] can be introduced to further increase the compressive strain in $\mathrm{GaSb}$.

\section{Conclusion}

We have introduced compressive strain in GaSb NWs along [111]-direction by epitaxially growing GaSb-GaAs $\mathrm{Sb}_{1-x}$ core-shell heterostructures integrated on $\mathrm{Si}$ substrates. Various shell As compositions and core-shell dimensions have been investigated to establish the growth conditions to accommodate the highest axial compressive strain in the GaSb core. The axial strain is obtained and its relaxation mechanism is described by a theoretical model for loop dislocations, also offering a guideline to achieve high strain in the GaSb core without dislocationinduced plastic relaxation. The hydrostatic strain is further determined and the strain effects on the band structure are evaluated, showing more benefits than existing biaxially strained GaSb films for hole mobility enhancement. Our results unlock the strain engineering in GaSb-based NWs and suggest that high compressive strain can also be introduced into GaSb NWs with high quality epitaxy, which provides an attractive option to further improve the electronic properties, in particular the hole mobility, of p-type MOSFETs to be used in for instance all-III-V CMOS digital applications.

\section{Methods}

\subsection{Nanowire growth}

Prior to the growth, large arrays with an area of $1.5 \mathrm{~mm} \times 3 \mathrm{~mm}$ consisting of Au discs with a pitch of $1 \mu \mathrm{m}$ and diameters of 20-36 nm were patterned by electron-beam lithography on $\mathrm{Si}$ (111) substrates with a 260-nm-thick InAs layer on top. All the NWs were grown by MOVPE in a close-coupled showerhead system 18313 at a pressure of 100 mbar and a total flow of $8,000 \mathrm{sccm}$. The growth is initialized with an InAs stem at $448{ }^{\circ} \mathrm{C}$ for 32 or $36 \mathrm{~nm} \mathrm{Au}$ particles while $465^{\circ} \mathrm{C}$ for $20 \mathrm{~nm}$ ones, using trimethylindium (TMIn) and arsine with a molar fraction of $X_{\mathrm{TMIn}}=6.25 \times 10^{-6}$ and $X_{\mathrm{AsH}_{3}}=1.25 \times 10^{-4}$, respectively. Next, GaSb was grown while heating up and mainly at $505^{\circ} \mathrm{C}$ with trimethylgallium (TMGa) and TMSb with a molar fraction of $X_{\mathrm{TMGa}}=4.9 \times 10^{-5}$ and $X_{\mathrm{TMSb}}=2.1 \times 10^{-4}$, respectively. At the same temperature, GaAsSb shells were finally grown with the same $X_{\mathrm{TMGa}}$ and a V/III ratio of 2 .

\subsection{TEM characterization}

The structural and compositional analysis using TEM consisted of HRTEM imaging in conventional TEM mode and spectroscopic acquisition using XEDS, the latter in STEM mode. The TEM used was a JEM-3000F, operated at $300 \mathrm{kV}$. NWs were transferred to lacy carbon covered Cu-grids by pressing the grid against the substrate, hence breaking off wires. HRTEM micrographs were acquired and used to analyze the local lattice and defects. Compositional mapping was performed in STEM mode using an XEDS-detector (Oxford Instruments) and quantified using the corresponding software Aztec.

\subsection{XRD measurements}

Lab-setup Bruker D8 system equipped with a $40 \mathrm{kV} \mathrm{Cu} \mathrm{X-ray}$ source $(\lambda=1.541 \AA)$ was used for all XRD measurements. All the NW samples with the large array of $1.5 \mathrm{~mm} \times 3 \mathrm{~mm}$ were directly measured in XRD setup. (111) $\omega / 2 \theta$ scans with large $\omega$-offset $\Delta \omega=0.8^{\circ}$ were carried out for probing the axial strain along NW growth direction.

\subsection{Raman measurements}

Renishaw inVia Raman microscopy equipped with Leica $\mathrm{N}$ Plan $(100 \times / 0.9)$ objective was used for strain study of individual NWs which were first transferred onto a pre-patterned $\mathrm{SiO}_{2}$ substrate coated with Au. All Raman spectra were measured with an excitation laser with a wavelength of $785 \mathrm{~nm}$. In all cases, the laser power and spot size were $\sim 0.1 \mathrm{~mW}$ and $\sim 2 \mu \mathrm{m}$, respectively.

\subsection{Theoretical modeling}

The finite-element continuum elasticity model included in COMSOL Multiphysics was used for strain field simulations of core-shell NWs. The lattice mismatch between GaSb and the $\mathrm{GaAs}_{x} \mathrm{Sb}_{1-x}$ shell was selected as the initial strain. A 3D model of NWs with finite length $(500 \mathrm{~nm})$ was built and the elastic constant matrix of materials were converted from $<001>$ to $<111>[22,54]$. Edge dislocation loops at the interface of the core and shell were intentionally taken into account to explain the mechanism of strain relaxation $[42,55]$. The Burgers vector $\boldsymbol{b}=a / 3$ [111] in the loop results in a reduced initial mismatch along the NW growth direction [42, 56]. Straincoupling band edge at the $\Gamma$-point of $\mathrm{GaSb}$ core was calculated by band-edge deformation potential theory [46]. GaSb band parameters along $<111>$ were extracted from Ref. [46].

\section{Acknowledgements}

This work was supported by the Swedish Research Council (VR), and the Swedish Foundation for Strategic Research (SSF).

Funding note: Open access funding provided by Lund University.

Electronic Supplementary Material: Supplementary material (details on the validation of the shell As composition and axial strain in the core, effects of the As gas composition, XRD and Raman results for all samples and FEM modeling with loop dislocations) is available in the online version of this article at https://doi.org/10.1007/s12274-020-2889-3.

Open Access This article is licensed under a Creative Commons Attribution 4.0 International License, which permits use, sharing, adaptation, distribution and reproduction in any medium or format, as long as you give appropriate credit to the original author(s) and the source, provide a link to the Creative Commons licence, and indicate if changes were made.

The images or other third party material in this article are included in the article's Creative Commons licence, unless indicated otherwise in a credit line to the material. If material is not included in the article's Creative Commons licence and your intended use is not permitted by statutory regulation or exceeds the permitted use, you will need to obtain permission directly from the copyright holder.

To view a copy of this licence, visit http://creativecommons.org/licenses/by/4.0/. 


\section{References}

[1] Tomioka, K.; Yoshimura, M.; Fukui, T. A III-V nanowire channel on silicon for high-performance vertical transistors. Nature 2012, 488, 189-192.

[2] Svensson, J.; Dey, A. W.; Jacobsson, D.; Wernersson, L. E. III-V nanowire complementary metal-oxide semiconductor transistors monolithically integrated on Si. Nano Lett. 2015, 15, 7898-7904.

[3] Kilpi, O. P.; Svensson, J.; Wu, J.; Persson, A. R.; Wallenberg, R.; Lind, E.; Wernersson, L. E. Vertical InAs/InGaAs heterostructure metal-oxide- semiconductor field-effect transistors on Si. Nano Lett. 2017, 17, 6006-6010.

[4] Jönsson, A.; Svensson, J.; Wernersson, L. E. A self-aligned gate-last process applied to all-III-V CMOS on Si. IEEE Electron Device Lett. 2018, 39, 935-938.

[5] Lynall, D.; Nair, S. V.; Gutstein, D.; Shik, A.; Savelyev, I. G.; Blumin, M.; Ruda, H. E. Surface state dynamics dictating transport in InAs nanowires. Nano Lett. 2018, 18, 1387-1395.

[6] del Alamo, J. A. Nanometre-scale electronics with III-V compound semiconductors. Nature 2011, 479, 317-323.

[7] Kilpi, O. P.; Svensson, J.; Lind, E.; Wernersson, L. E. Electrical properties of vertical InAs/InGaAs heterostructure MOSFETs. IEEE J. Electron Devices Soc. 2019, 7, 70-75.

[8] Dey, A. W.; Svensson, J.; Borg, B. M.; Ek, M.; Wernersson, L. E. Single InAs/GaSb nanowire low-power CMOS inverter. Nano Lett. 2012, 12, 5593-5597.

[9] Oktyabrsky, S. P-type channel field-effect transistors. In Fundamentals of III-V Semiconductor MOSFETs. Oktyabrsky, S.; Ye, P., Eds.; Springer: Boston, MA, 2010; pp 349-378.

[10] Borg, M.; Schmid, H.; Gooth, J.; Rossell, M. D.; Cutaia, D.; Knoedler, M.; Bologna, N.; Wirths, S.; Moselund, K. E.; Riel, H. High-mobility GaSb nanostructures cointegrated with InAs on Si. ACS Nano 2017, $11,2554-2560$.

[11] Yang, Z. X.; Yip, S.; Li, D. P.; Han, N.; Dong, G. F.; Liang, X. G.; Shu, L.; Hung, T. F.; Mo, X. L.; Ho, J. C. Approaching the hole mobility limit of GaSb nanowires. ACS Nano 2015, 9, 9268-9275.

[12] Yang, Z. X.; Liu, L. Z.; Yip, S.; Li, D. P.; Shen, L. F.; Zhou, Z. Y.; Han, N.; Hung, T. F.; Pun, E. Y. B.; Wu, X. L. et al. Complementary metal oxide semiconductor-compatible, high-mobility, $\langle 111\rangle$-oriented $\mathrm{GaSb}$ nanowires enabled by vapor-solid-solid chemical vapor deposition. ACS Nano 2017, 11, 4237-4246.

[13] Sun, J. M.; Peng, M.; Zhang, Y. S.; Zhang, L.; Peng, R.; Miao, C. C.; Liu, D.; Han, M. M.; Feng, R. F.; Ma, Y. D. et al. Ultrahigh hole mobility of Sn-catalyzed $\mathrm{GaSb}$ nanowires for high speed infrared photodetectors. Nano Lett. 2019, 19, 5920-5929.

[14] Chen, Y. W.; Tan, Z.; Zhao, L. F.; Wang, J.; Liu, Y. Z.; Si, C.; Yuan, F.; Duan, W. H.; Xu, J. Mobility enhancement of strained GaSb p-channel metal-oxide-semiconductor field-effect transistors with biaxial compressive strain. Chin. Phys. B 2016, 25, 038504.

[15] Bennett, B. R.; Ancona, M. G.; Boos, J. B.; Canedy, C. B.; Khan, S. A. Strained GaSb/AlAsSb quantum wells for p-channel field-effect transistors. J. Cryst. Growth 2008, 311, 47-53.

[16] Bennett, B. R.; Chick, T. F.; Ancona, M. G.; Brad Boos, J. Enhanced hole mobility and density in GaSb quantum wells. Solid State Electron. 2013, 79, 274-280.

[17] Borg, B. M.; Dick, K. A.; Ganjipour, B.; Pistol, M. E.; Wernersson, L. E.; Thelander, C. InAs/GaSb heterostructure nanowires for tunnel field-effect transistors. Nano Lett. 2010, 10, 4080-4085.

[18] Wang, X. Y.; Du, W. N.; Yang, X. G.; Zhang, X. W.; Yang, T. Self-catalyzed growth mechanism of InAs nanowires and growth of InAs/GaSb heterostructured nanowires on Si substrates. J. Cryst. Growth 2015, 426, 287-292.

[19] Jeppsson, M.; Dick, K. A.; Wagner, J. B.; Caroff, P.; Deppert, K.; Samuelson, L.; Wernersson, L. E. GaAs/GaSb nanowire heterostructures grown by MOVPE. J. Cryst. Growth 2008, 310, 4115-4121.

[20] Zamani, R. R.; Gorji Ghalamestani, S.; Niu, J.; Sköld, N.; Dick, K. A. Polarity and growth directions in $\mathrm{Sn}$-seeded $\mathrm{GaSb}$ nanowires. Nanoscale 2017, 9, 3159-3168.

[21] Ye, H.; Lu, P. F.; Yu, Z. Y.; Song, Y. X.; Wang, D. L.; Wang, S. M. Critical thickness and radius for axial heterostructure nanowires using finite-element method. Nano Lett. 2009, 9, 1921-1925.

[22] Grönqvist, J.; Søndergaard, N.; Boxberg, F.; Guhr, T.; Åberg, S.;
$\mathrm{Xu}, \mathrm{H}$. Q. Strain in semiconductor core-shell nanowires. J. Appl. Phys. 2009, 106, 053508.

[23] Glas, F. Strain in nanowires and nanowire heterostructures. In Semiconductors and Semimetals. Morral, A. F. I.; Dayeh, S. A.; Jagadish, C., Eds.; Elsevier: United States, 2015; pp 79-123.

[24] Lewis, R. B.; Nicolai, L.; Küpers, H.; Ramsteiner, M.; Trampert, A.; Geelhaar, L. Anomalous strain relaxation in core-shell nanowire heterostructures via simultaneous coherent and incoherent growth. Nano Lett. 2017, 17, 136-142.

[25] Balaghi, L.; Bussone, G.; Grifone, R.; Hübner, R.; Grenzer, J.; Ghorbani-Asl, M.; Krasheninnikov, A. V.; Schneider, H.; Helm, M.; Dimakis, E. Widely tunable GaAs bandgap via strain engineering in core/shell nanowires with large lattice mismatch. Nat. Commun. 2019, 10, 2793.

[26] Göransson, D. J. O.; Borgström, M. T.; Huang, Y. Q.; Messing, M. E.; Hessman, D.; Buyanova, I. A.; Chen, W. M.; Xu, H. Q. Measurements of strain and bandgap of coherently epitaxially grown wurtzite InAsP-InP core-shell nanowires. Nano Lett. 2019, 19, 2674-2681.

[27] Lazarev, S.; Göransson, D. J. O.; Borgström, M.; Messing, M. E.; Xu, H. Q.; Dzhigaev, D.; Yefanov, O. M.; Bauer, S.; Baumbach, T.; Feidenhans'l, R. et al. Revealing misfit dislocations in $\operatorname{InAs}{ }_{x} \mathrm{P}_{1-x}-\mathrm{InP}$ core-shell nanowires by X-ray diffraction. Nanotechnology 2019, 30,505703 .

[28] Sun, Y.; Thompson, S. E.; Nishida, T. Physics of strain effects in semiconductors and metal-oxide-semiconductor field-effect transistors. J. Appl. Phys. 2007, 101, 104503.

[29] Sun, Y. K.; Thompson, S. E.; Nishida, T. Strain Effect in Semiconductors: Theory and Device Applications; Springer: Boston, MA, 2010.

[30] Gluschke, J. G.; Leijnse, M.; Ganjipour, B.; Dick, K. A.; Linke, H.; Thelander, C. Characterization of ambipolar GaSb/InAs core-shell nanowires by thermovoltage measurements. ACS Nano 2015, 9 , 7033-7040.

[31] Vasen, T.; Ramvall, P.; Afzalian, A.; Doornbos, G.; Holland, M.; Thelander, C.; Dick, K. A.; Wernersson, L. E.; Passlack, M. Vertical gate-all-around nanowire GaSb-InAs core-shell n-type tunnel FETs. Sci. Rep. 2019, 9, 202.

[32] Ganjipour, B.; Ek, M.; Mattias Borg, B.; Dick, K. A.; Pistol, M. E.; Wernersson, L. E.; Thelander, C. Carrier control and transport modulation in $\mathrm{GaSb} / \mathrm{InAsSb}$ core/shell nanowires. Appl. Phys. Lett. 2012, 101, 103501.

[33] Salehzadeh, O.; Kavanagh, K. L.; Watkins, S. P. Growth and strain relaxation of GaAs and $\mathrm{GaP}$ nanowires with $\mathrm{GaSb}$ shells. J. Appl. Phys. 2013, 113, 134309.

[34] Montazeri, M.; Fickenscher, M.; Smith, L. M.; Jackson, H. E.; Yarrison-Rice, J.; Kang, J. H.; Gao, Q.; Tan, H. H.; Jagadish, C.; Guo, Y. et al. Direct measure of strain and electronic structure in GaAs/GaP core-shell nanowires. Nano Lett. 2010, 10, 880-886.

[35] Ek, M.; Borg, B. M.; Johansson, J.; Dick, K. A. Diameter limitation in growth of III-Sb-containing nanowire heterostructures. ACS Nano 2013, 7, 3668-3675.

[36] Yang, Z. X.; Han, N.; Fang, M.; Lin, H.; Cheung, H. Y.; Yip, S.; Wang, E. J.; Hung, T.; Wong, C. Y.; Ho, J. C. Surfactant-assisted chemical vapour deposition of high-performance small-diameter GaSb nanowires. Nat. Commun. 2014, 5, 5249.

[37] Namazi, L.; Nilsson, M.; Lehmann, S.; Thelander, C.; Dick, K. A. Selective GaSb radial growth on crystal phase engineered InAs nanowires. Nanoscale 2015, 7, 10472-10481.

[38] Borg, B. M.; Dick, K. A.; Eymery, J.; Wernersson, L. E. Enhanced $\mathrm{Sb}$ incorporation in InAsSb nanowires grown by metalorganic vapor phase epitaxy. Appl. Phys. Lett. 2011, 98, 113104.

[39] Koblmüller, G.; Hertenberger, S.; Vizbaras, K.; Bichler, M.; Bao, F.; Zhang, J. P.; Abstreiter, G. Self-induced growth of vertical freestanding InAs nanowires on $\mathrm{Si}(111)$ by molecular beam epitaxy. Nanotechnology 2010, 21, 365602.

[40] Çakan, A.; Sevik, C.; Bulutay, C. Strained band edge characteristics from hybrid density functional theory and empirical pseudopotentials: GaAs, GaSb, InAs and InSb. J. Phys. D: Appl. Phys. 2016, 49, 085104.

[41] Dayeh, S. A.; Tang, W.; Boioli, F.; Kavanagh, K. L.; Zheng, H.; Wang, J.; Mack, N. H.; Swadener, G.; Huang, J. Y.; Miglio, L. et al. 
Direct measurement of coherency limits for strain relaxation in heteroepitaxial core/shell nanowires. Nano Lett. 2013, 13, 1869-1876.

[42] Raychaudhuri, S.; Yu, E. T. Critical dimensions in coherently strained coaxial nanowire heterostructures. J. Appl. Phys. 2006, 99, 114308 .

[43] Aoki, K.; Anastassakis, E.; Cardona, M. Dependence of Raman frequencies and scattering intensities on pressure in GaSb, InAs, and InSb semiconductors. Phys. Rev. B 1984, 30, 681-687.

[44] Arora, A. K.; Rajalakshmi, M.; Ravindran, T. R.; Sivasubramanian, V. Raman spectroscopy of optical phonon confinement in nanostructured materials. J. Raman Spectrosc. 2007, 38, 604-617.

[45] Cerdeira, F.; Buchenauer, C. J.; Pollak, F. H.; Cardona, M. Stressinduced shifts of first-order Raman frequencies of diamond- and zinc-blende-type semiconductors. Phys. Rev. B 1972, 5, 580-593.

[46] Van de Walle, C. G. Band lineups and deformation potentials in the model-solid theory. Phys. Rev. B 1989, 39, 1871-1883.

[47] Li, S. S. Scattering mechanisms and carrier mobilities in semiconductors. In Semiconductor Physical Electronics; Li, S. S., Ed.; Springer: New York, 2006; pp 211-245.

[48] Silver, M.; Batty, W.; Ghiti, A.; O’Reilly, E. P. Strain-induced valence-subband splitting in III-V semiconductors. Phys. Rev. B 1992, 46, 6781-6788.

[49] Nainani, A.; Kim, D.; Krishnamohan, T.; Saraswat, K. Hole mobility and its enhancement with strain for technologically relevant III-V semiconductors. In 2009 International Conference on Simulation of
Semiconductor Processes and Devices, San Diego, CA, USA, 2009, pp 1-4.

[50] Thompson, S.; Sun, G.; Wu, K.; Lim, J.; Nishida, T. Key differences for process-induced uniaxial vs. Substrate-induced biaxial stressed $\mathrm{Si}$ and Ge channel MOSFETs. In IEDM Technical Digest. IEEE International Electron Devices Meeting, 2004. San Francisco, CA, USA, 2004, pp 221-224.

[51] Goldthorpe, I. A.; Marshall, A. F.; McIntyre, P. C. Synthesis and strain relaxation of Ge-core/Si-shell nanowire arrays. Nano Lett. 2008, 8, 4081-4086.

[52] Hashemi, P.; Gomez, L.; Canonico, M.; Hoyt, J. L. Electron transport in gate-all-around uniaxial tensile strained-Si nanowire n-MOSFETs. In 2008 IEEE International Electron Devices Meeting, San Francisco, CA, USA, 2008, pp 1-4.

[53] Signorello, G.; Karg, S.; Björk, M. T.; Gotsmann, B.; Riel, H. Tuning the light emission from GaAs nanowires over $290 \mathrm{meV}$ with uniaxial strain. Nano Lett. 2013, 13, 917-924.

[54] Boxberg, F.; Søndergaard, N.; Xu, H. Q. Elastic and piezoelectric properties of zincblende and wurtzite crystalline nanowire heterostructures. Adv. Mater. 2012, 24, 4692-4706.

[55] Kavanagh, K. L. Misfit dislocations in nanowire heterostructures. Semicond. Sci. Technol. 2010, 25, 024006.

[56] Biermanns, A.; Rieger, T.; Bussone, G.; Pietsch, U.; Grützmacher, D.; Ion Lepsa, M. Axial strain in GaAs/InAs core-shell nanowires. Appl. Phys. Lett. 2013, 102, 043109. 